九州大学学術情報リポジトリ

Kyushu University Institutional Repository

\title{
Aeroacoustic simulation of broadband sound generated from low-Mach-number flows using a lattice Boltzmann method
}

Kusano, Kazuya

Hitachi, Ltd., Research \& Development Group

山田，和豊

Department of Systems Innovation Engineering, Iwate University

古川，雅人

九州大学大学院工学研究院機械工学部門

http://hdl. hand le. net/2324/4481545

出版情報：Journal of Sound and Vibration. 467 (115044)，2020-02-17. Elsevier バージョン：

権利関係 : 


\title{
Aeroacoustic simulation of broadband sound generated from low-Mach-number flows using a lattice Boltzmann method
}

\author{
Kazuya Kusano ${ }^{\mathrm{a}, \star}$, Kazutoyo Yamada ${ }^{\mathrm{b}}$, and Masato Furukawa ${ }^{\mathrm{c}}$ \\ ${ }^{a}$ Hitachi, Ltd., Research \& Development Group, 832-2, Horiguchi, Hitachinaka, Ibaraki, 312-0034, Japan \\ ${ }^{\mathrm{b}}$ Department of Systems Innovation Engineering, Iwate University, 4-3-5, Ueda, Morioka, 020-8551, Japan \\ c Department of Mechanical Engineering, Kyushu University, 744 Motooka, Nishi-ku, Fukuoka, 819-0395, Japan \\ * Corresponding author. E-mail address: kazuya.kusano.bn@hitachi.com
}

\begin{abstract}
The present paper demonstrates the capability of a numerical method based on the lattice Boltzmann method (LBM) with wall-resolved grid to predict the broadband sound generated from the turbulent boundary layer at low Mach numbers. The present method is based on the lattice BGK equation with the D2Q9 and D3Q15 models, and a multi-scale approach using hierarchically refined grids is proposed to efficiently and simultaneously capture the multi-scale phenomena of turbulent eddies near walls and far-field sound waves. Numerical instabilities caused by the lack of grid resolution are suppressed with a fourth-order implicit filtering scheme. This numerical method is discussed in two benchmark problems and an application to the prediction of the broadband sound generated from the turbulent boundary layer. First, the computational accuracy and speed of the LBM scheme are assessed with a pulse-propagating problem. The results indicate that the LBM can achieve accuracy comparable to the fourth-order central scheme with the four-stage Runge-Kutta method for the compressible Navier-Stokes (N-S) equations and compute 12.3 times faster. These findings suggest that the LBM is an efficient computational method for aeroacoustic simulations. Second, the proposed method is validated by simulating the Aeolian tone generated by the flow past a circular cylinder at Reynolds number of 150 and Mach number of 0.2 . The present simulation is compared with a compressible N-S simulation using a high-order finite difference scheme in terms of the wave profile and the propagation speed of the tonal sound. This validation result suggests that the present method is available for direct aeroacoustic simulations of low-Mach-number flows. Finally, the capability of the present method to predict the broadband sound is demonstrated by conducting a wall-resolved simulation for the turbulent flow generated by a short separation bubble over an isolated airfoil at Reynolds number of $2.0 \times 10^{5}$ and Mach number of 0.058 . This simulation shows a good agreement with measurements of the surface pressure distributions, the wake velocity profiles, and the far-field sound spectrum. In contrast to hybrid approaches based on the incompressible N-S equations, the present method can accurately predict the broadband sound in the high-frequency range by simulating the acoustic scattering on the airfoil.
\end{abstract}




\section{Keywords}

Computational aeroacoustics, Lattice Boltzmann method, Cartesian grid, Broadband sound, Turbulent flow

\section{Nomenclature}

$\mathbf{x}$ position

$t \quad$ time

$\mathbf{c}_{i} \quad$ particle velocity

$f_{i} \quad$ particle distribution function

$f_{i}^{\text {eq }} \quad$ local equilibrium distribution function

$\tau \quad$ relaxation time

$\rho \quad$ fluid density

u fluid velocity

$p \quad$ fluid pressure

$v \quad$ fluid dynamic viscosity

$c_{s} \quad$ sound speed

$\hat{f}_{i} \quad$ distribution function after the collision operation

$\tilde{f}_{i} \quad$ distribution function after the filtering operation

D cylinder diameter

$C_{p} \quad$ pressure coefficient

$C_{D} \quad$ drag coefficient

$C_{L} \quad$ lift coefficient

C airfoil chord length

Re Reynolds number

Ma Mach number

St Strouhal number 


\section{Introduction}

The prediction of the sound induced by low-Mach-number flows is important in the aerodynamic design of low-speed fans, which are installed in home appliances, information equipment and, vehicles. In previous studies on fan noise, numerical predictions have been achieved for the aerodynamic sounds associated with large flow structures such as wakes, distorded inflows, and tip vortices, which are detached from walls $[1,2,3]$. On the other hand, the prediction of the broadband sound generated from the turbulent flow attached to blade surfaces remains a challenging problem for actual applications.

To predict the sounds induced by low-Mach-number flows, several hybrid approaches have been developed over the past two decades [4, 5]. In these hybrid approaches, the incompressible NavierStokes (N-S) equations is solved by several approaches such as the large eddy simulation (LES) to obtain time series of pressure fluctuation relating to unsteady flows, and the far-field sound is calculated from the incompressible N-S results on the basis of Lighthill's acoustic analogy [6]. The most practical choice for acoustic calculations is Curle's equation [7], but the pressure fluctuations obtained from the incompressible N-S equations cannot be used for calculating such integral solutions if the object is not sufficiently compact compared with the wavelength of the radiated sound. To predict the high-frequency broadband sound generated from the attached turbulent flow, it is important to consider the scattering of the sounds radiated from turbulence eddies on walls by the compressible flow simulation. The conventional density-based schemes for the compressible N-S equations, however, suffer the stiff problem and the severe Courant-Freidrichs-Lewy (CFL) condition in computing low-Mach-number flows [8].

Recently, the lattice Boltzmann method (LBM) has attracted great attention as an alternative approach for aeroacoustic simulations of low-Mach-number flows because of its computational efficiency $[9,10,11]$. The tonal and broadband sounds generated from the flow past tandem cylinders have successfully predicted using a commercial solver based on the LBM and the Ffowcs WilliamsHawkings (FW-H) equation [12]. In the literature, these sounds are induced by the largely separated flow, which has been simulated using the $\mathrm{k}-\varepsilon$ turbulence model with a wall function on a zonal refinement grid. This approach has been applied to many applications such as the trailing-edge noise of an airfoil [13], the broadband noise of a turbofan with outlet guide vanes [14], and the vortex-blade interaction noise of a helicopter [15]. On the other hand, only a few studies have attempted a wallresolved simulation with the LBM [16], which is effective to the prediction of the broadband sound generated from the attached turbulent. In such a simulation, regular lattices should be efficiently arranged across the computational domain to simultaneously capture both turbulent eddies near walls and far-field sound waves at feasible computational cost.

The purpose of this study was to demonstrate the capability of the numerical method based on the LBM to predict the broadband sound generated from attached turbulent flows at low Mach numbers. A multi-scale approach using hierarchically refined grids adapted to object geometries was proposed 
to efficiently and simultaneously capture the multi-scale phenomena of turbulent eddies near walls and far-field sound waves. In the method, numerical instabilities arising in coarse grids are suppressed with a filtering operation. The proposed method was tested with two benchmark problems and applied to the prediction of the broadband sound generated from an attached turbulent flow. First, the computational accuracy and speed of the LBM scheme were investigated in a pulse-propagating problem. Second, the present numerical method was validated for the Aeolian tone generated by the flow past a circular cylinder at Reynolds number of 150 and Mach number of 0.2 in comparison with a high-order finite difference scheme for the compressible N-S equations. Finally, the broadband sound of the turbulent flow generated by a short separation bubble over an isolated airfoil at Reynolds number of $2.0 \times 10^{5}$ and Mach number of 0.058 was predicted with a wall-resolved simulation, and the simulation results were compared with measurement data in detail.

\section{Numerical method}

\subsection{Lattice Boltzmann method}

In the LBM, flow and sound are simulated by computing the lattice Boltzmann equation, which is a kinetic equation that describes the streaming and collision processes of particles with a finite number of velocities. The lattice Boltzmann equation with the Bhatnagar-Gross-Krook (BGK) collision model $[17,18]$ is written as

$$
f_{i}\left(t+\Delta t, \mathbf{x}+\Delta t \mathbf{c}_{i}\right)=f_{i}(t, \mathbf{x})-\frac{1}{\tau}\left[f_{i}(t, \mathbf{x})-f_{i}^{\mathrm{eq}}(t, \mathbf{x})\right],
$$

where $f_{i}(t, \mathbf{x})$ is the distribution function of a particle with velocity $\mathbf{c}_{i}$ at position $\mathbf{x}$ and time $t$. The right-hand side of Eq. (1) is the collision operator, which assumes that the particles relax to the local equilibrium states represented by $f_{i}^{\text {eq }}$ at a relaxation time $\tau$. This collision operator can be computed locally and is suitable for parallel computing. Furthermore, the streaming operation can be completed only by shifting the distribution function from one node to its neighbor, since the time increment $\Delta t$ satisfies the relation $\Delta \mathbf{x}=\Delta t \mathbf{c}_{i}$, where $\Delta \mathbf{x}$ is the grid spacing. That means the CFL number is enforced to be 1 .

The fluid density $\rho$ and velocity $\mathbf{u}$ are defined as follows:

$$
\rho=\sum_{i} f_{i}, \quad \rho \mathbf{u}=\sum_{i} f_{i} \mathbf{c}_{i} .
$$

As discrete velocity models, we adopted the D2Q9 and the D3Q15 athermal models [18], which consider the conservation of mass and momentum only. In these models, the local equilibrium velocity distribution function $f_{i}^{\text {eq }}$ is given by

$$
f_{i}^{\mathrm{eq}}=w_{i} \rho\left[1+3 \mathbf{c}_{i} \cdot \mathbf{u}+\frac{9}{2}\left(\mathbf{c}_{i} \cdot \mathbf{u}\right)^{2}-\frac{3}{2} \mathbf{u} \cdot \mathbf{u}\right]
$$

Here $w_{i}$ is the weighting factor, which is defined as $w_{0}=4 / 9, w_{1 \sim 4}=1 / 9, w_{5 \sim 8}=1 / 36$ in the 
D2Q9 and $w_{0}=2 / 9, w_{1 \sim 6}=1 / 9, w_{7 \sim 14}=1 / 72$ in the D3Q15.

The compressible Navier-Stokes equations are derived from the models using the ChapmannEnskog expansion, and the relaxation time $\tau$ is related to the fluid dynamic viscosity $v$ as follows:

$$
\tau=\frac{1}{2}+\frac{3 v}{\Delta t c^{2}}
$$

Moreover, in the athermal models, the fluid pressure is related to the density as $p=\rho c_{s}^{2}$, where $c_{s}$ is the speed of sound. The speed of sound is constant at $c_{s}=c / \sqrt{3}$. All simulations presented below were performed for air at temperature of $293 \mathrm{~K}$, and $v=1.5 \times 10^{-5} \mathrm{~m}^{2} / \mathrm{s}$ and $c_{s}=343 \mathrm{~m} / \mathrm{s}$ were used. As reported in the previous studies $[9,11,19]$, the present study assumes that the athermal models are applicable to aeroacoustic simulations of weakly compressible low-Mach-number flows [20, 21], since temperature change is negligible there.

\subsection{Multi-scale approach}

The LBM model restricts the lattice shape to the uniform Cartesian grid. This subsection proposes a multi-scale approach using locally refined grids to overcome this difficulty and simulate turbulent eddies near walls and far-field sound waves at feasible computational cost.

The present approach adopts the Building-Cube method (BCM) proposed by Nakahashi and Kim [22] to generate the locally refined grids. The BCM recursively divides a cubic domain that contains wall boundaries into eight sub-domains. All cubes in the bottom layer are discretized into the uniform Cartesian grids with a same number of grid points. This process generates a hierarchically refined grid adapted to object geometries. In the present multi-scale approach, the LBM computation is performed for each cube under each relaxation time, while communicating with neighboring cubes.

The computational procedure at the interface between two differently sized cubes is illustrated in Fig. 1. The lower cube is two times finer than the upper one, and nodes of the both grids overlap at the interface. After the collision operation, the spatial and temporal interpolations are implemented for the distribution functions in the coarse grid. Then, before the streaming operation, the distribution function for each grid is transformed to that for the neighboring grid using a rescaling scheme.

The rescaling scheme is derived to ensure the continuity of the density, the momentum and their derivatives at the interface between different resolution grids as follows [23, 24]:

$$
\begin{aligned}
& \hat{f}_{i}^{\mathcal{F}}=f_{i}^{\mathrm{eq}, \mathcal{C}}-\frac{1}{\Omega}\left(\hat{f}_{i}^{\mathcal{C}}-f_{i}^{\mathrm{eq}, \mathcal{C}}\right) \\
& \hat{f}_{i}^{\mathcal{C}}=f_{i}^{\mathrm{eq}, \mathcal{F}}-\Omega\left(\hat{f}_{i}^{\mathcal{F}}-f_{i}^{\mathrm{eq}, \mathcal{F}}\right) .
\end{aligned}
$$

The superscripts $\mathcal{F}$ and $\mathcal{C}$ mark functions and parameters in the fine and coarse grids, respectively. $\hat{f}$ is the distribution function after the collision operation. The parameter $\Omega$ is defined as follows: 


$$
\Omega=n \frac{\tau^{\mathcal{C}}-1}{\tau^{\mathcal{F}}-1},
$$

where $n$ is the ratio of coarse to fine grid spacing, and it is fixed to two in the present grid hierarchy.

In the present study, the computations were parallelized based on the domain decomposition, by which neighboring cubes were allocated to the same core.

\subsection{Filtering operation}

Our application of the LBM with the BGK model suffers from numerical instabilities caused by the low grid resolution in some of the hierarchically defined cubes, since the relaxation time is a small value in these regions. To suppress the numerical instabilities arising in coarse grids, a filtering operation was incorporated into the LBM scheme. The filtering operation was implemented for the distribution function using the following implicit scheme [25].

$$
\begin{aligned}
a_{f} \widetilde{f}_{l}\left(t, \mathbf{x}-\mathbf{c}_{i} \Delta t\right) & +\widetilde{f}_{l}(t, \mathbf{x})+a_{f} \widetilde{f}_{l}\left(t, \mathbf{x}+\mathbf{c}_{i} \Delta t\right) \\
& =\sum_{n=0}^{N} \frac{a_{n}}{2}\left\{f_{i}\left(t, \mathbf{x}-n \mathbf{c}_{i} \Delta t\right)+f_{i}\left(t, \mathbf{x}+n \mathbf{c}_{i} \Delta t\right)\right\},
\end{aligned}
$$

where the fourth-order formula $(N=2)$ was adopted, and the free parameter $a_{f}$ was set to 0.45 .

The cubes that contain wall boundaries are sufficiently refined to maintain the numerical stability in the original operations, and this filtering operation was not applied to such cubes.

\subsection{Wall boundary condition}

To accurately represent arbitrary geometries with a Cartesian grid, the interpolated bounce-back scheme [26] was used for the wall boundary condition. Figure 2 illustrates this scheme for a direction $i$ of discrete velocities. The black dots indicate computational nodes, where $\mathbf{x}_{1}$ is the closest node to the wall in the fluid, $\mathbf{x}_{2}$ is the node adjacent to $\mathbf{x}_{1}$ toward the fluid, and $\mathbf{x}_{s}$ is the neighbor on the solid side. The white dot indicates a fictitious node, which is located so that a particle starting from the fictitious node just reaches $\mathbf{x}_{1}$ in one time step. The distribution function at the fictitious node is estimated and given to $\mathbf{x}_{S}$ as a boundary condition as follows:

$$
\hat{f}_{\bar{l}}\left(t, \mathbf{x}_{s}\right)=\left\{\begin{array}{ll}
\frac{2 q_{i}}{\Delta x} \hat{f}_{i}\left(t, \mathbf{x}_{1}\right)+\frac{\left(\Delta x-2 q_{i}\right)}{\Delta x} \hat{f}_{i}\left(t, \mathbf{x}_{2}\right) & \left(q_{i}<\Delta x / 2\right) \\
\frac{\Delta x}{2 q_{i}} \hat{f}_{i}\left(t, \mathbf{x}_{1}\right)+\frac{\left(2 q_{i}-\Delta x\right)}{2 q_{i}} \hat{f}_{\bar{l}}\left(t, \mathbf{x}_{1}\right) & \left(q_{i} \geq \Delta x / 2\right)
\end{array},\right.
$$

where $\bar{l}$ denotes the opposite direction to $i$. The scheme is applied to all the directions of discrete velocities for the particle model used, and thereby it can accurately represent the boundary of arbitrary geometries in both two and three dimensional simulations. 


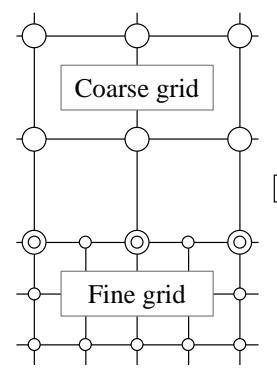

Collision

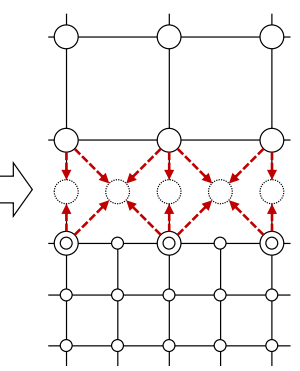

Interpolation

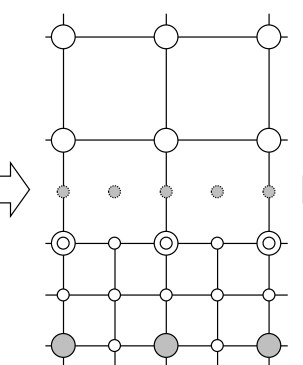

Rescaling

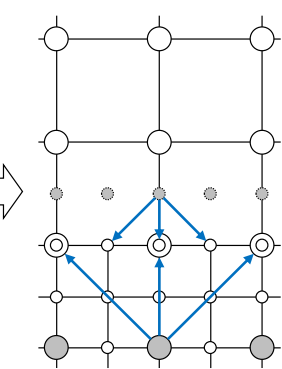

Streaming

Fig. 1. Computational procedure at the interface between two differently sized cubes.

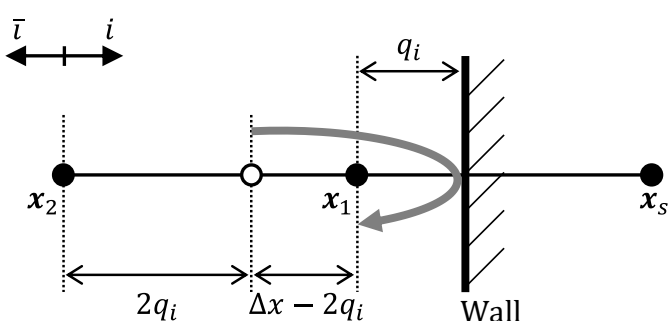

(a)

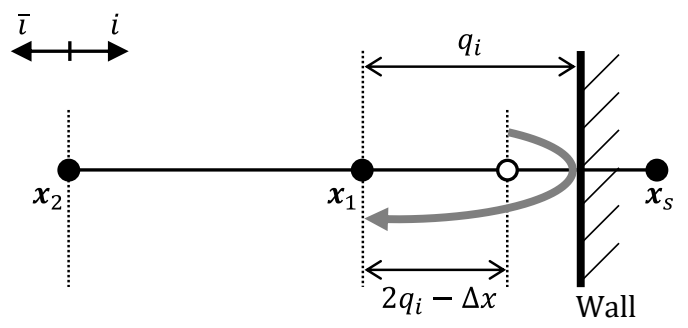

(b)

Fig. 2. Illustration of the interpolated bounce-back scheme. (a) $q_{i}<\Delta x / 2$; (b) $q_{i} \geq \Delta x / 2$.

\subsection{Outer boundary condition and absorbing region}

At the outer boundaries of the computational domain, free-stream conditions were employed with the local equilibrium functions calculated from the free-stream density and velocity. The function is indicated by $f_{i}^{\infty}$.

Moreover, to prevent sound waves from reflecting off the outer boundaries, sound waves were absorbed in a specified region by adding the following damping term to Eq. (1) [27]:

$$
f_{i}\left(t+\Delta t, \mathbf{x}+\Delta t \mathbf{c}_{i}\right)=f_{i}(t, \mathbf{x})-\frac{1}{\tau}\left[f_{i}(t, \mathbf{x})-f_{i}^{\mathrm{eq}}(t, \mathbf{x})\right]-\alpha\left(\frac{x_{d}}{L_{d}}\right)^{2}\left[f_{i}^{\mathrm{eq}}(t, \mathbf{x})-f_{i}^{\infty}(t, \mathbf{x})\right],
$$

where $L_{d}$ indicates the width of the absorbing region in the radial direction, and $x_{d}$ indicates the distance from the inner boundary of the absorbing region. The parameter $\alpha$ was set to $\alpha=0.05$.

\section{Benchmark problems}

This section tests the present method with basic aeroacoustic problems before applying it to the prediction of the broadband sound. In the problems, the present method are compared with the conventional finite difference method (FDM) for the compressible N-S equations in terms of the computational accuracy and speed, and the availability of the present method for direct aeroacoustic simulations of low-Mach-number flows are discussed. 


\subsection{Propagation of Gaussian pulse}

This subsection compares the LBM with the finite differencing schemes for solving the compressible N-S equations in terms of the computational accuracy and speed for a two dimensional pulse-propagation problem.

The athermal LBM with the D2Q9 velocity model and three finite differencing schemes for the compressible N-S equations are considered. One is the second-order central spatial differencing scheme with the two-stage Runge-Kutta method for time integration (E2RK2), and the second is the explicit fourth-order central scheme with the four-stage Runge-Kutta method (E4RK4). The third scheme is the sixth-order compact scheme [28] in combination with the four-stage Runge-Kutta method (C6RK4).

Benchmark computations were performed for a Gaussian pulse propagating in a uniform flow. The computational domain extending $-10 \mathrm{~m} \leq x \leq 10 \mathrm{~m},-10 \mathrm{~m} \leq y \leq 10 \mathrm{~m}$ was discretized in the three type of grids. Grid I and Grid II are uniform with resolutions $\Delta x=0.1 \mathrm{~m}$ and $\Delta x=0.05 \mathrm{~m}$, respectively. Grid III consists of two resolutions, which are $\Delta x=0.05 \mathrm{~m}$ in $-5 \mathrm{~m} \leq x \leq$ $5 \mathrm{~m},-5 \mathrm{~m} \leq y \leq 5 \mathrm{~m}$ and $\Delta x=0.1 \mathrm{~m}$ in the other region. The uniform flow at a Mach number of 0.1 was set along the $x$ axis. The initial density and velocity was given as follows:

$$
\rho(x, y, 0)=\rho_{\infty}+\varepsilon \exp \left[-(\ln 2)\left(x^{2}+y^{2}\right) / b\right], \quad \mathrm{u}=u_{\infty}, \quad \mathrm{v}=0,
$$

where $\varepsilon=1.0 \times 10^{-5}$ and $b=0.1$. The initial temperature was uniform at $293 \mathrm{~K}$. The analytical solution of the linearized Euler equations for this initial value problem is [29]:

$$
\rho^{\prime}(x, y, t)=\frac{\varepsilon}{2 \alpha} \int_{0}^{\infty} \exp \left[-\xi^{2} / 4 \alpha\right] \cos (\xi t) J_{0}(\xi \eta) \xi \mathrm{d} \xi
$$

where $\alpha=\ln 2 / b, \eta=\sqrt{(x-\mathrm{Mat})^{2}+y^{2}}$, and $J_{0}$ is the Bessel function of the first kind and order zero. The CFL number was enforced to 1 in the LBM as mentioned above, and that was specified to 0.6 in the FDMs. The computations were completed before the pulse reached the outer boundary of the domain.

Figure 3 exhibits the instantaneous distributions of the pressure fluctuation on the $x$ axis at $t=$ $0.0176 \mathrm{~s}$ for the computational results and the analytical solution. Figure 3 (a) compares the results among different schemes, where Grid I $(\Delta x=0.1 \mathrm{~m})$ was used. The curves between the lower and upper peaks are resolved with approximately 7 nodes in Grid I. Obvious differences among the schemes appear in Fig. 3 (a). Although both of the LBM and the E2RK2 have second-order accuracy in theory [20, 30], Fig. 3 (a) shows that the LBM is less dispersive than the E2RK2. This result is consistent with that of Marie et al. [9]. Moreover, Fig. 3 (a) shows that the LBM is comparable to the E4RK4 and inferior to the C6RK4 in accuracy. The C6RK4 result agreed well with the analytical solution. Figure 3 (b) compares the LBM results among different grids. The LBM result using Grid II $(\Delta x=0.05 \mathrm{~m})$ almost agreed with the analytical solution. The LBM therefore can provide solutions comparable to the C6RK4 by using a double resolution grid. In Grid III with multi-resolution, there is 
a discontinuity of resolution at $x=5 \mathrm{~m}$, but significant influences are not observed in the result of Fig. 3 (b). These results indicate that the error caused by the resolution discontinuity is relatively small.

Figure 4 shows the amplitude $A$ and phase $\varphi$ of the pressure fluctuation at $x=5 \mathrm{~m}, \mathrm{y}=0 \mathrm{~m}$ in the frequency domain by the ratio to the analytical solution. Obviously, the LBM shows a smaller dispersion error than the E2RK2 and the comparable accuracy to the E4RK4.

Table 1 shows the computational times per one grid point per one time step measured for the schemes by the ratio to the LBM. These computations were performed without parallel processing. The LBM is 12.3 times faster than the E4RK4, which gave a comparable solution with that of the LBM in this benchmark test, and the LBM is 16.3 times faster than the C6RK4. Although the LBM is inferior to the C6RK4 in accuracy, this inaccuracy can be compensated for by increasing the grid resolution.

The computational cost per one grid point per one time step for the LBM simulations is proportional to the number of the discrete velocities, and the D3Q15 requires 15/9 times more computational time than the D2Q9. The computational cost for the three-dimensional compressible NS simulation using a FDM scheme is estimated to be 15/8 times higher than that for the twodimensional simulation with the same scheme by comparing the number of equations and the number of special differential terms. These estimated values are comparable, so it seems feasible to apply the above discussion on the two-dimensional simulations to the three-dimensional simulations.

These findings suggest that the LBM is an efficient computational method for aeroacoustic simulations. Also note that the LBM refers only to adjacent nodes, thus the LBM could compute much more efficiently than the high-order differencing schemes in parallel computations based on domaindecomposition techniques.

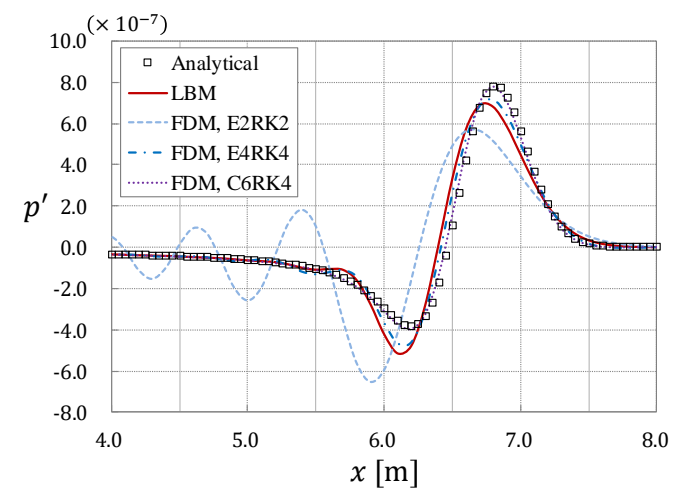

(a)

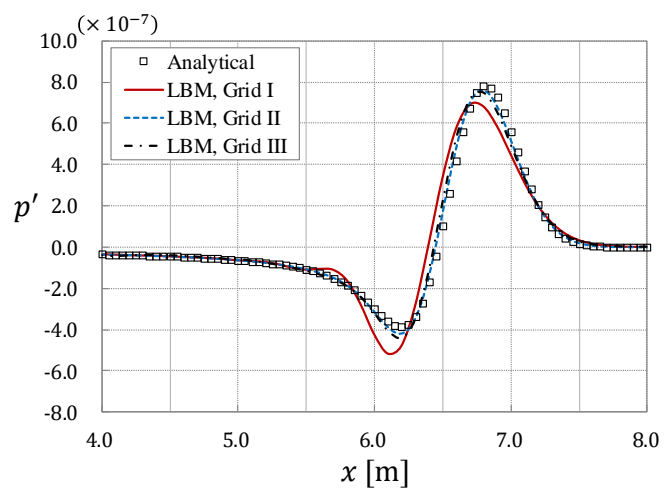

(b)

Fig. 3. Instantaneous distributions of the pressure fluctuation on the $\mathrm{x}$ axis at $t=0.0176 \mathrm{~s}$. (a) Different schemes, with Grid I; (b) different grids, using the LBM. 


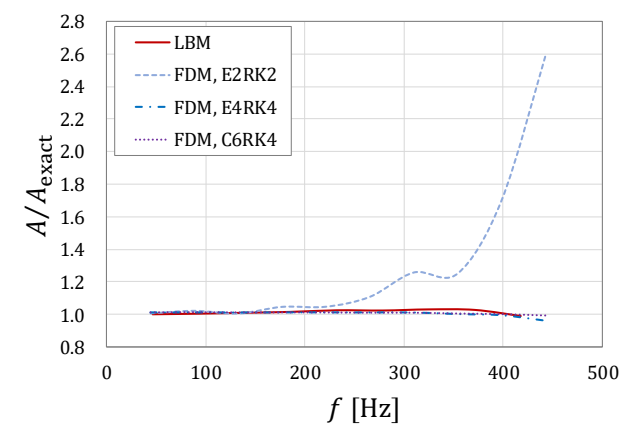

(a)

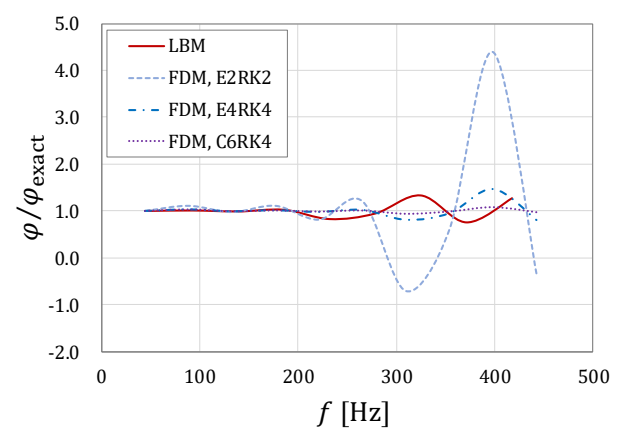

(b)

Fig. 4. Amplitude and phase of the pressure fluctuation at $x=5 \mathrm{~m}, \mathrm{y}=0 \mathrm{~m}$ in the frequency domain.

(a) Amplitude; (b) phase.

Table 1 Computational times measured for the schemes indicated by the ratio to the LBM.

\begin{tabular}{|c||c|c|c|c|}
\hline Scheme & LBM & E2RK2 & E4RK4 & C6RK4 \\
\hline Computational time & 1.0 & 6.0 & 12.3 & 16.3 \\
\hline
\end{tabular}

\subsection{Aeolian tone of a circular cylinder}

Next, the Aeolian tone generated by the flow past a circular cylinder was computed in two dimensions. In this subsection, the computed flow and acoustic fields are compared between the present method and the high-order FDM for the compressible N-S equations on a body-fitted structure grid reported by Inoue and Hatakeyama [31].

The Reynolds number based on the cylinder diameter $D$ was set to 150 . The cylinder diameter $D$ is $3.3 \times 10^{-5} \mathrm{~m}$. At this Reynolds number, the Karman vortex street is formed in the wake behind the circular cylinder, and the wake is laminar [32]. The free-stream Mach number was set to 0.2. This Mach number is a bit high for the LBM, but the simulation was successfully conducted without any special treatment. The computational domain is a square which extends over the area of $-1024 D \leq$ $x \leq 1024 D,-1024 D \leq y \leq 1024 D$, where $\mathrm{x}$ and $\mathrm{y}$ denote the coordinates parallel and normal to the free stream, respectively. The center of the circular cylinder coincides with the origin.

Figure 5 shows the computational domain, which is divided into 628 sub-domains by the BCM. Each sub-domain includes $129^{2}$ grid points, and the total number of grid points is 10.5 million. The smallest grid spacing around the cylinder surface is $0.000977 \mathrm{D}$, and the largest grid spacing around the outer boundaries is $2 D$. This grid resolves the wavelength of the tonal sound with at least 14 grid points. As shown in Fig. 5, the absorbing region is defined as $r>768 \mathrm{D}$, where $r$ is the radial coordinate $\left(r=x^{2}+y^{2}\right)$. The width of the absorbing region $L_{d}$ is $256 D$, which is sufficiently large to include nine wavelengths or more in the radial direction.

First, the validation results for the flow fields will be discussed. Figure 6 plots the time-averaged and instantaneous pressure distributions on the cylinder surface, where the azimuth angle $\theta$ is defined 
so that $\theta=180^{\circ}$ coincides with the streamwise direction. The plots show smooth pressure distributions, and the time-averaged pressure agrees well with the result obtained from the FDM simulation with a body-fitted grid [31]. These results show the validity of the present treatment for wall boundaries using the interpolated bounce-back scheme. Figure 7 shows the time variations of the drag and lift coefficients. Time $t$ is normalized with the cylinder diameter and the free-stream velocity, where normalized time $t=-10$ and $t=10$ corresponds to 4024000 and 4202000 time steps, respectively. The drag and lift coefficients were calculated by approximating the cylinder surface using the nearest nodes in the Cartesian grid. This approximation may account for the underestimation of the drag coefficient that can be seen in Fig. 7. The amplitudes and periods of the drag and lift fluctuations, which are induced by Karman vortices, are consistent in the LBM and FDM results. The Strouhal number for the lift fluctuation is 0.183 , which is equal to that reported by Inoue and Hatakeyama [31]. These results show that the present method accurately captures the fluctuations of the fluid force that contribute to the tonal sound as a dipole sound source.

Next, the validation results for acoustic fields are discussed. Figure 8 shows instantaneous contours of pressure fluctuations. In Fig. 8, (a) and (b) display the near and far fields, respectively, and the black lines indicate the boundaries of the computational blocks. Figure 8 shows that the strongest sounds are radiated from the cylinder in the direction nearly normal to the streamwise direction, due to the large fluctuation of the lift. In Fig. 8, the contour lines are smoothly drawn across the blocks with different resolutions, and non-physically generated or distorted sounds are not observed at the block interfaces. These results indicate that the multi-scale approach is applicable to direct aeroacoustic simulations. Furthermore, Fig. 9 shows the RMS of the pressure fluctuation along the radial direction $\left(\theta=90^{\circ}\right)$. Figure 9 compares the LBM results with and without the absorbing region. The LBM results is consistent with the decay law of $r^{-1 / 2}$ at positions sufficiently far away from the cylinder. In the result without the absorbing region, standing waves are observed near the outer boundary. On the other hand, in the result with the absorbing region, the pressure fluctuation is sufficiently damped near the outer boundary. Figure 10 shows instantaneous distributions of pressure fluctuations along the radial direction $\left(\theta=90^{\circ}\right)$ at three time instants $(t=3.3,4.3$, and 5.3). Consistently, the results of the LBM are in excellent agreement with those of the FDM. This means that the sound simulated with the athermal LBM propagates at the same speed as the compressible N-S simulation, although the energy conservation law is not explicitly imposed on the athermal LBM. Figure 11 shows power spectral densities of pressure fluctuations at three positions. Frequency $f$ is normalized with the tone frequency $f_{\text {tone. }}$. In the spectrum at the position of $r=D$, peaks are identified only at the tone and its second and third harmonic frequencies, and no spurious sound is generated near the cylinder. On the other hand, spurious sounds are observed in the high-frequency range at the positions of $r=10 \mathrm{D}$ and $r=100 D$, but these sounds are much smaller than the tone.

The present simulation agreed well with the high-order finite difference scheme for the 
compressible Navier-Stokes equations in terms of the wave profile and the propagation speed of the tonal sound. Although this test is for a low-Reynolds-number flow with a simple geometry, these results show that the athermal model and the multi-scale approach can be used for direct aeroacoustic simulations of low-Mach-number flows.

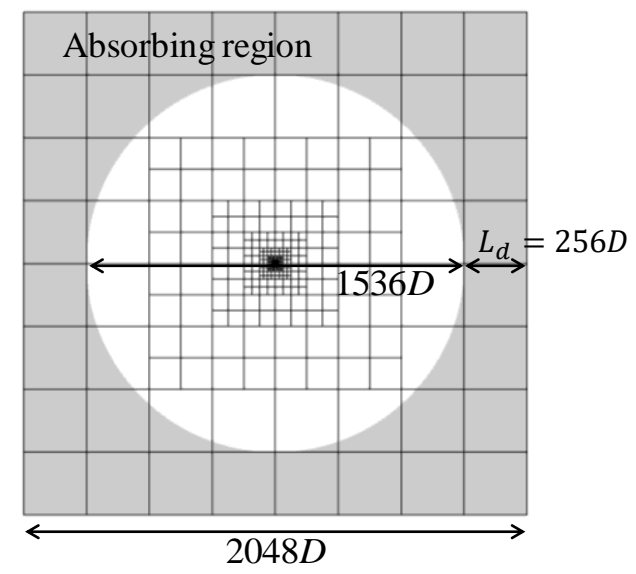

Fig. 5. Computational domain for the circular cylinder's Aeolian tone.

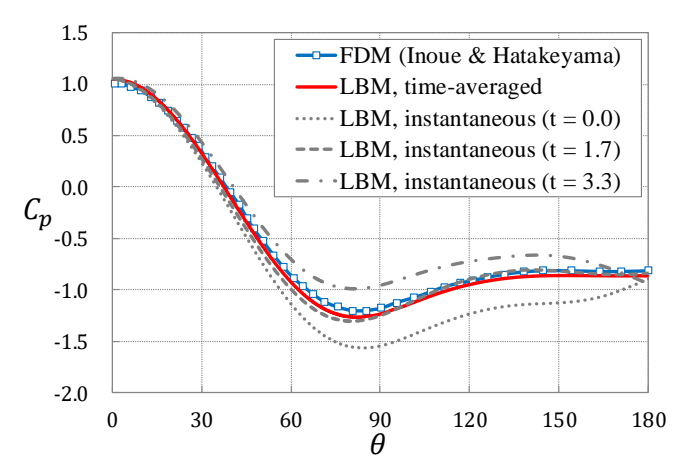

Fig. 6. Time-averaged and instantaneous pressure coefficient distributions on the cylinder surface.

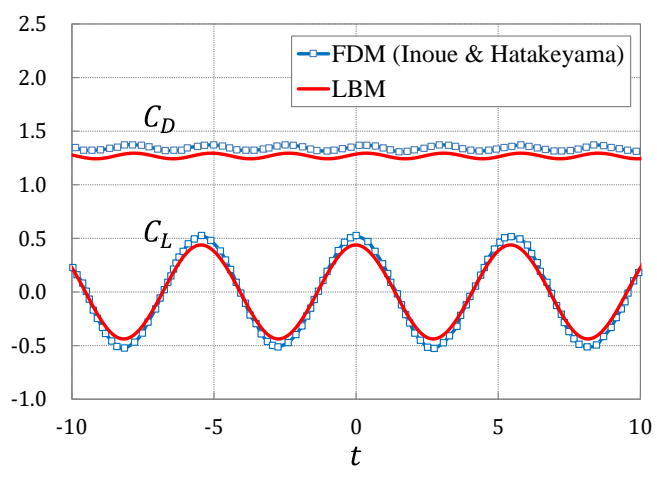

Fig. 7. Time variations of drag and lift coefficients. 


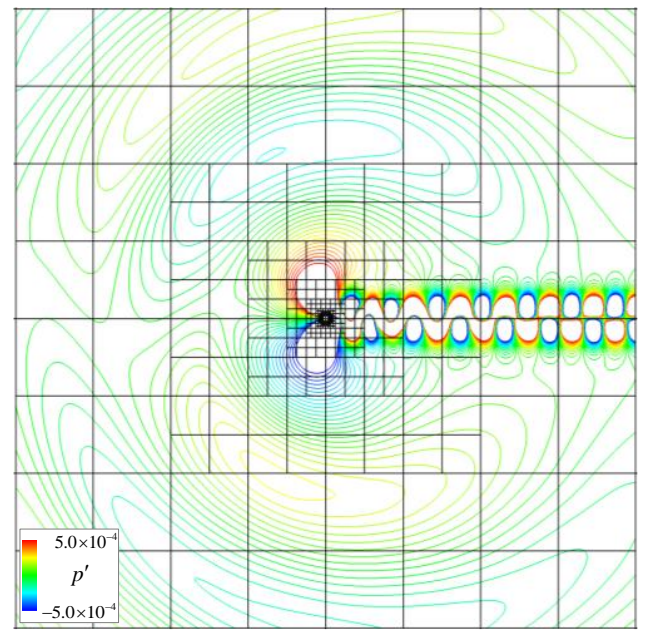

(a)

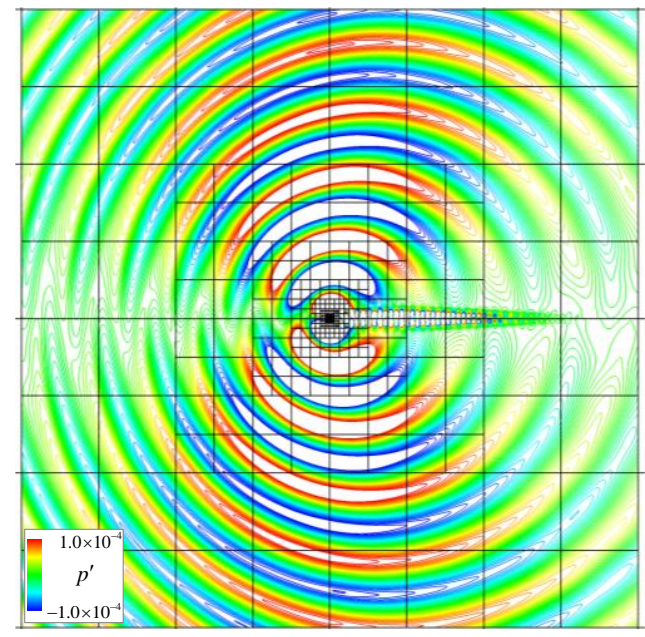

(b)

Fig. 8. Instantaneous distributions of pressure fluctuation. (a) $-32 D \leq x \leq 32 D,-32 D \leq y \leq 32 D$; (b) $-128 D \leq x \leq 128 D,-128 D \leq y \leq 128 D$.

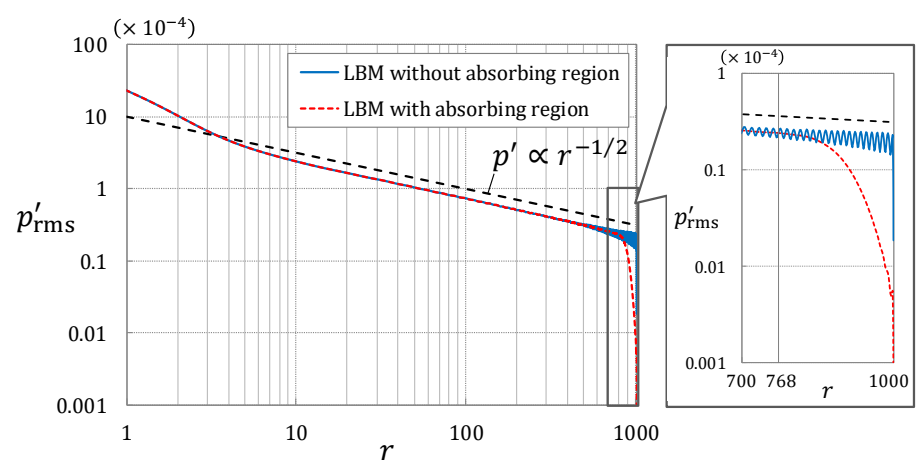

Fig. 9. RMS of pressure fluctuation along the radial direction $\left(\theta=90^{\circ}\right)$.

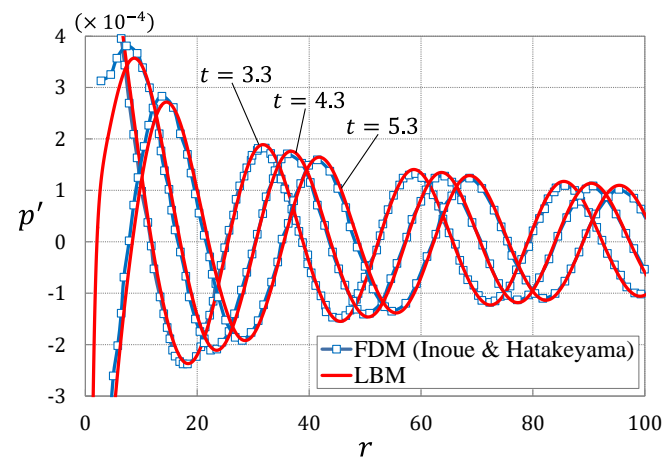

Fig. 10. Instantaneous distributions of pressure fluctuation along the radial direction $\left(\theta=90^{\circ}\right)$ at three time instants.

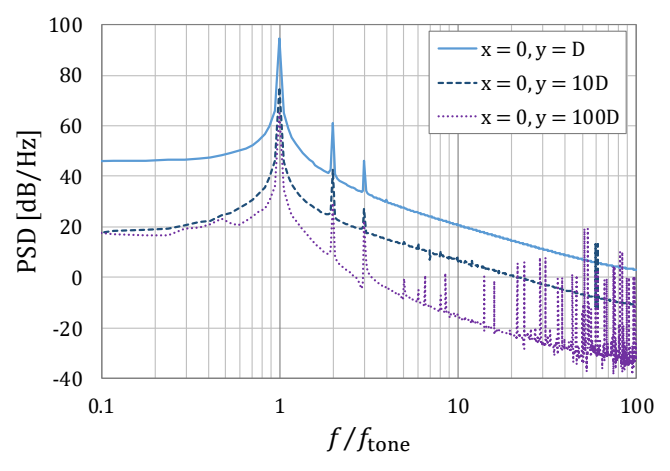

Fig. 11. Power spectral densities of pressure fluctuation at three positions. 


\section{Broadband sound of an isolated airfoil}

\subsection{Computational setup}

This section discusses the simulation results on the broadband sound generated from the turbulent flow around an isolated airfoil. The computational results will be compared in detail with experimental results reported by Kato et al. [5] and Miyazawa et al. [33].

In the simulations, a NACA0012 airfoil was placed in a uniform flow. The airfoil chord length $C$ is $0.15 \mathrm{~m}$, and the free-stream Mach number is 0.058 , and the angle of attack is $9.5^{\circ}$. The flow Reynolds number based on the chord length is $2.0 \times 10^{5}$. Under these conditions, the flow around the airfoil is turbulent downstream of a leading-edge separation, and the flow-induced sound shows a broadband spectrum.

The computational domain extended over the area of $-12 C \leq x \leq 12 C,-12 C \leq y \leq 12 C, 0 \leq$ $z \leq 0.125 C$, where $x, y$, and $z$, respectively, denote the coordinates in the chordwise direction, the normal direction to the chord, and the spanwise direction. This domain size is sufficiently large for the sound prediction as shown in Appendix A. The leading edge of the airfoil was placed at the position of $x=0, y=0$. The span length of the airfoil is $0.125 C$, which is sufficiently large to contain several streak structures in the turbulent boundary layer on the airfoil. In the spanwise direction, the periodic boundary condition was employed. To prevent sound waves from reflecting on the outer boundaries of the domain, an absorbing region was set at $r>8 C$, where $r$ is the radial coordinate $\left(r=x^{2}+\right.$ $y^{2}$ ). The width of the absorbing region $L_{d}$ is $4 C$.

The computational domain is divided into approximately 240,000 cubic blocks, and each block has $17^{3}$ nodes, for a total of about 1.2 billion grid points. The maximum grid spacing around the outer boundaries is $0.0078 \mathrm{C}$. This grid can resolve a sound wave of frequency $15 \mathrm{kHz}$ with at least 20 grid points. Figure 12 shows the distribution of blocks around the airfoil. The grid was designed to resolve the turbulent boundary layer on the airfoil directly. The minimum grid spacing around the airfoil is $2.4 \times 10^{-4} C$, which is 2.3 in terms of wall units. In addition to this fine grid, a coarse grid, which is a half resolution of the fine grid in all directions, was used to investigate the sensitivity of the numerical method to grid resolution. The total number of grid points in the coarse grid is about 0.3 billion. Table 1 lists the grid information used for the wall resolved simulations of an isolated NACA0012 airfoil in the literature [5, 34-37] and in the present study. The grid resolutions in the streamwise and spanwise directions of the fine grid used for the present study are the finest of those listed in Table 1. On the other hand, the wall-normal grid spacing of the fine grid used for the present study is comparable to that used by Kato et al. and Marsden et al. [5, 34], and is relatively coarser than the others. This is one of the first attempts of applying a wall-resolved simulation using the LBM with a Cartesian grid to the turbulent flow around an airfoil.

In the case of the fine grid, the value of the relaxation time ranges from 0.50006 to 0.50210 . Generally, the standard BGK model cannot perform stable computations under such small values of 
the relaxation time. In the present study, the filtering operation enabled such computations by suppressing the numerical instabilities.

The outer boundary condition was given by $f_{i}^{\infty}$, which is the local equilibrium functions calculated from the free-stream density and velocity. Also, $f_{i}^{\infty}$ was used for the initial condition.

The computation using the fine grid was performed with 3200 cores of Fujitsu PRIMERGY CX400 and was completed in $8.0 \times 10^{6}$ time steps, which were needed to fully develop the flow and acquire the statistical data for post-processing. The total computational time was approximately 770 hours.

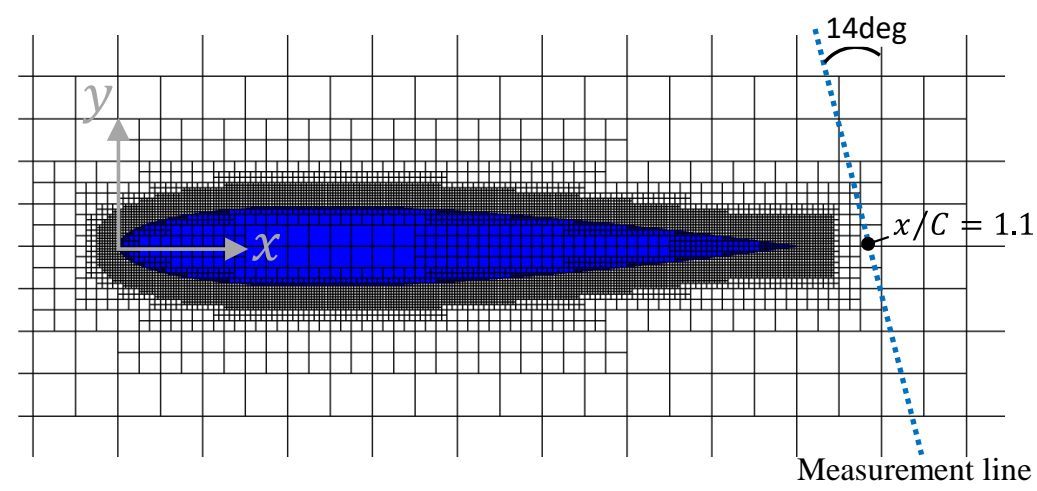

Fig. 12. Computational domain divided into cubic blocks around the NACA0012 airfoil.

Table 2. Grid information for wall-resolved simulations of turbulent flows around a NACA0012 airfoil.

\begin{tabular}{|l|c|c|c|c|c|c|c|}
\hline Author & Year & Method & Grid type & Re & $\Delta \boldsymbol{x}^{+}$ & $\Delta \boldsymbol{y}^{+}$ & $\Delta \boldsymbol{z}^{+}$ \\
\hline Kato et al. [5] & 2007 & FEM & Body-fitted & $2.0 \times 10^{5}$ & 50 & 2 & 50 \\
\hline Marsden et al. [34] & 2008 & FDM & Body-fitted & $5.0 \times 10^{5}$ & 20 & 2.5 & 20 \\
\hline Shan et al. [35] & 2008 & FDM & Body-fitted & $1.0 \times 10^{5}$ & 18 & 0.75 & 6 \\
\hline Wolf et al. [36] & 2012 & FDM & Body-fitted & $4.08 \times 10^{5}$ & 50 & 0.5 & 20 \\
\hline Zhang and Samtaney [37] & 2016 & FDM & Body-fitted & $5.0 \times 10^{4}$ & 5.6 & 0.9 & 10.8 \\
\hline Present (fine) & - & LBM & Cartesian & $2.0 \times 10^{5}$ & 2.3 & 2.3 & 2.3 \\
\hline Present (coarse) & - & LBM & Cartesian & $2.0 \times 10^{5}$ & 4.6 & 4.6 & 4.6 \\
\hline
\end{tabular}




\subsection{Results and discussion}

First, flow features around the airfoil will be discussed using the computational results with the fine grid. Figure 13 exhibits an instantaneous distribution of vorticity magnitude around the airfoil. This figure shows that the separated shear layer that appears over the suction surface near the leading edge transitions to the turbulent flow and subsequently reattaches to the airfoil surface. Downstream of the reattachment point, small eddies are resolved in the turbulent boundary layer.

Figure 14 displays time-averaged streamlines and a streamwise velocity contour around the suction surface near the leading edge. This time-averaged flow is consistent with the flow structure of the short separation bubble as reported by Horton $[38,39]$. The reattachment point is identified to be $x / C=$ 0.105, where the skin-friction coefficient is zero. In Fig. 14, white lines mark the computational blocks, and these confirm that the whole region of the separation bubble is computed with the finest grid resolution.

Figure 15 visualizes instantaneous flow fields in the boundary layer on the suction surface. Figure 15 (a) shows a pressure fluctuation contour on the suction surface, which is involved with generating the dipole sound. In Fig. 15 (a), the negative pressure fluctuation regions observed at $0.05<\mathrm{x} / C<$ 0.10 correspond to the spanwise vortices generated in the separated shear layer. These twodimensional vortices break down into multiple flow structures aligned in the spanwise direction and lead to large pressure fluctuations around the reattachment point $(x / C=0.105)$. Downstream of the reattachment point, a random distribution of the pressure fluctuation involved with turbulent flow is observed. Figure 15 (b) shows the contour of the streamwise velocity fluctuation near the suction surface $\left(y^{+} \approx 5\right)$. This figure shows several streaky flow structures, which represent the presence of longitudinal vortex, appearing at the downstream of the reattachment point $(x / C>0.105)$, and suggests that the present simulation with the fine mesh successfully captures the longitudinal vortices that appear in the turbulent boundary layer.

Next, the computed flow fields around the airfoil will be compared with experimental results. Figure 16 plots the time-averaged pressure coefficients along the airfoil surfaces. The pressure on the suction surface computed using the fine grid shows a plateau in the separation region $(0<\mathrm{x} / C<$ $0.10)$ and a rapid decrease at the reattachment point $(x / C=0.10)$. The computed reattachment point is shifted slightly downstream compared to the experimental one, and this shift may be caused by the shortage of grid resolution in the simulation or the presence of the free-stream turbulence in the experiment. Except for this difference, the simulation results for the fine grid are in good agreement with the experimental results [33]. In contrast, the coarse grid fails to capture these features.

Subsequently, Fig. 17 shows profiles of the time-averaged velocity and RMS velocity fluctuation in the wake obtained from the simulation using the fine grid and the measurement [33]. The traverse line of the velocity measurement passes through $\mathrm{x} / C=1.1, \mathrm{y} / C=0.0$ and inclines by 14 degrees to the $y$ axis as shown in Fig. 12. In the time-averaged profiles, the simulation is in excellent agreement 
with the experimental result. As for the RMS profiles, the simulation and measurement results show the blunt and sharp peaks on the suction side and the pressure side, respectively. The quantitative differences in these peaks can be attributed to the rapid decrease in the grid resolution near the traverse line as shown in Fig. 12.

Figure 18 shows the power spectrum densities (PSDs) of the pressure coefficient at $\mathrm{x} / C=$ $0.075,0.10,0.25$, and 0.90 on the suction surface obtained from the simulation using the fine grid and the measurement [33]. For the simulation results, the PSDs are averaged in the spanwise direction. It is reported that the peaks at $60<\mathrm{St}<100$ in the experimental results are attributed to the Helmholtz resonance of static pressure holes [33]. In Fig. 18 (a), the underestimation in the simulation at $\mathrm{x} / C=$ 0.075 is caused by the delay of the reattachment point in the simulation, and the simulation result at the position of $\mathrm{x} / C=0.10$, which is the reattachment point in the simulation, is closer to the experimental result at $\mathrm{x} / C=0.075$. At $\mathrm{x} / C=0.25$ and 0.90 , the simulation results are in good agreement with the experimental results. These validation results suggest that the sources of the broadband sound are reproduced with the wall-resolved simulation using the LBM.

Finally, the computed results of acoustic fields will be discussed. Figure 19 shows an instantaneous distribution of the pressure fluctuation in a $x-y$ plane obtained from the simulation using the fine grid. In Fig. 19, sound waves around the airfoil are shown as multiple concentric patterns with unequal spacing. This distribution indicates that the sound waves are radiating at multiple frequencies, and it is presumed that these sound waves are generated from the turbulent flow over the airfoil. Moreover, this figure shows that several wavelengths are comparably to the chord length of the airfoil. To accurately predict these high-frequency sounds, it is important to simulate the acoustic scattering on the airfoil as pointed out by Kato et al. [5]. This requirement can be achieved by the present method instead of the conventional incompressible N-S simulation for solving low-Mach-number flows.

Figure 20 shows the frequency spectra of the far-field sound observed at a position of $6.7 C$ away from the airfoil in the vertical direction. For the calculation of the sound spectra, the pressure history were collected over $1.1 \times 10^{6}$ time steps, which corresponds to $0.66 \mathrm{~s}$ in physical time. To obtain a smooth distribution, spectra calculated for 40 subsets of the data were averaged, where the frequency resolution is $61 \mathrm{~Hz}$. The computed sound pressure levels (SPLs) were corrected with respect to span length using the method shown in Appendix B. The simulation using the coarse grid, which failed to capture the separated and transitional flow around the airfoil, underestimated the SPL in the highfrequency range from 600 to $1100 \mathrm{~Hz}$. On the other hand, the simulation result for the fine grid, which successfully captured the flow around the airfoil, are in good agreement with the experimental result [5] in the high-frequency range $(f>700 \mathrm{~Hz})$. Large discrepancies appear in the low-frequency range between the simulation result for the fine grid and the experimental result. It is assumed that these discrepancies result from the facts that the present study did not model experimental circumstances aside from the airfoil itself. Generally, long wavelength sounds interact strongly with wind-tunnel 
components such as the nozzle and end plates.

The proposed method based on the LBM with the wall-resolved grid captured the turbulent flow generated by a short separation bubble over the airfoil. Furthermore, the method simulated the acoustic scattering of high-frequency sounds generated from the turbulent flow on the airfoil. Consequently, the method predicted the broadband sound spectrum including high-frequency components fairly well.

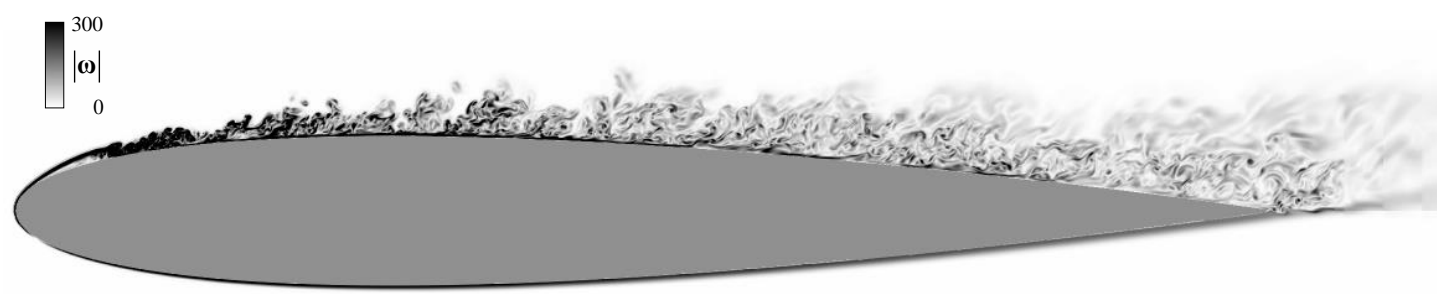

Fig. 13. Instantaneous distribution of vorticity magnitude around the airfoil.

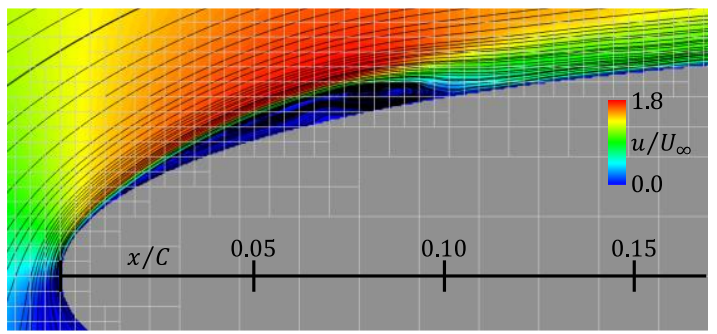

Fig. 14. Time-averaged streamlines and streamwise velocity contour on the suction surface near the leading edge.

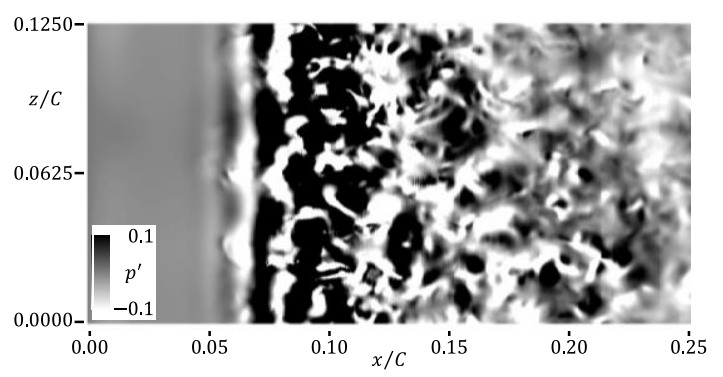

(a)

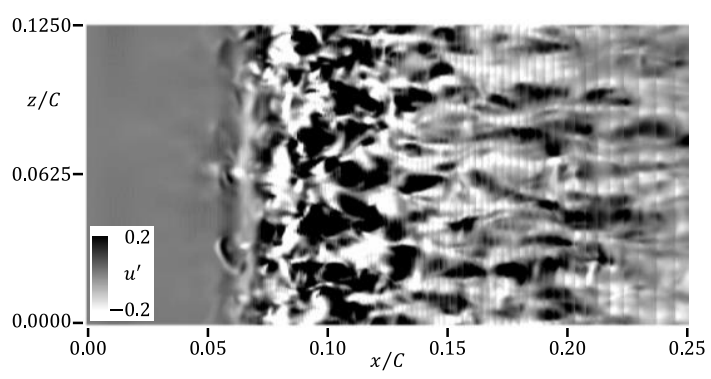

(b)

Fig. 15. Visualization of instantaneous flow structures in the boundary layer on the suction surface.

(a) Pressure fluctuation; (b) streamwise velocity fluctuation. 


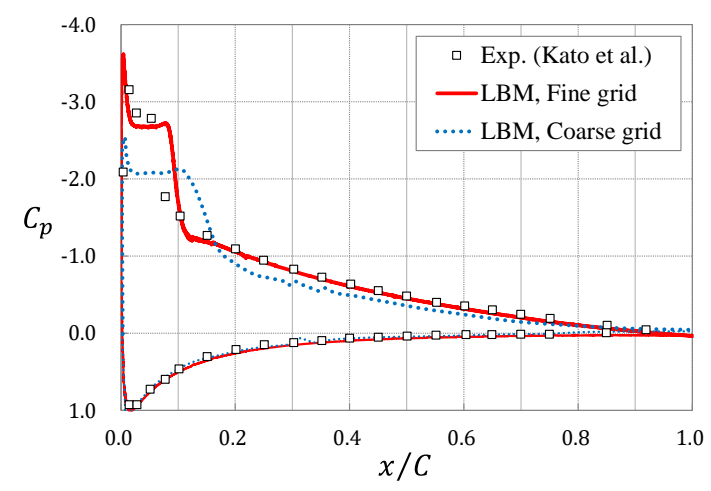

Fig. 16. Time-averaged pressure coefficients around the airfoil.

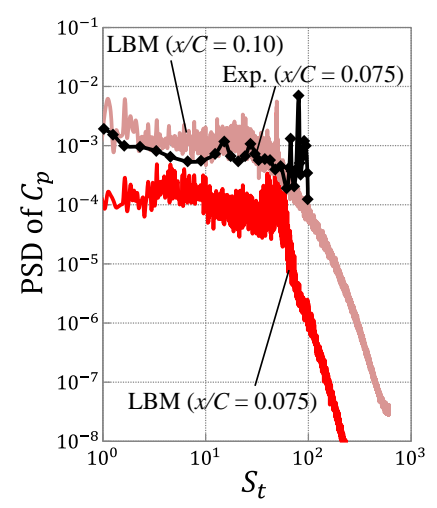

(a)

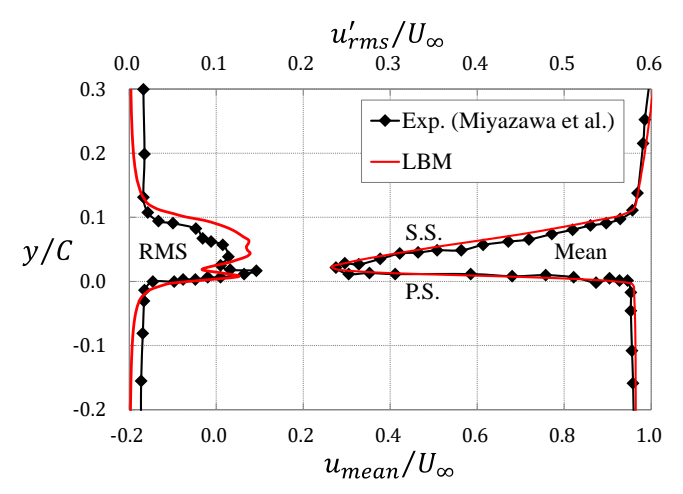

Fig. 17. Time-averaged velocity and RMS velocity profiles in the wake.

Fig. 18. Power spectrum densities of the pressure coefficients on the suction surface.

(a) $x / C=0.075$ and 0.10 ; (b) $x / C=0.25$; (c) $x / C=0.90$.

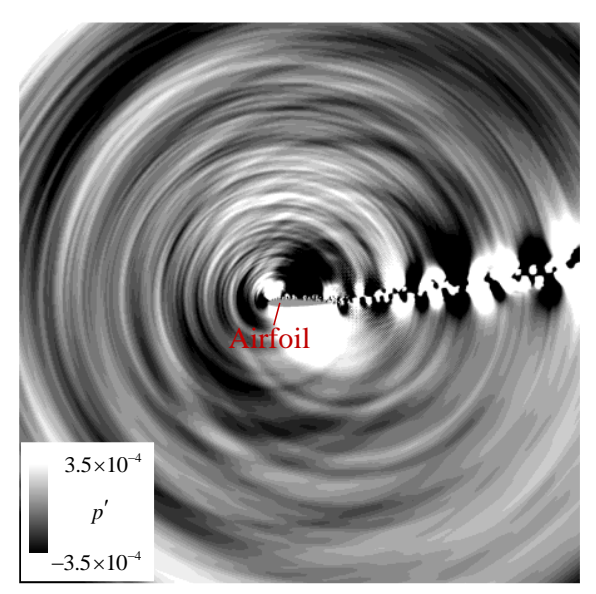

Fig. 19. Instantaneous distribution of pressure

fluctuation.

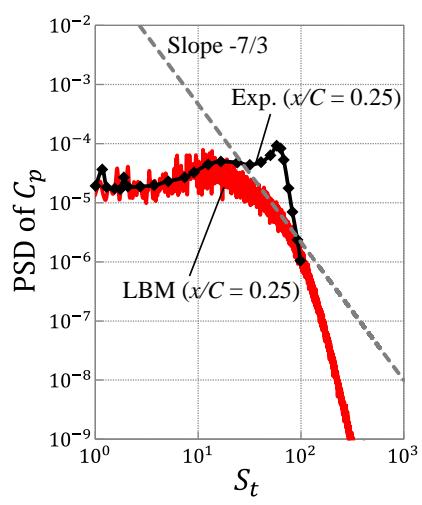

(b)

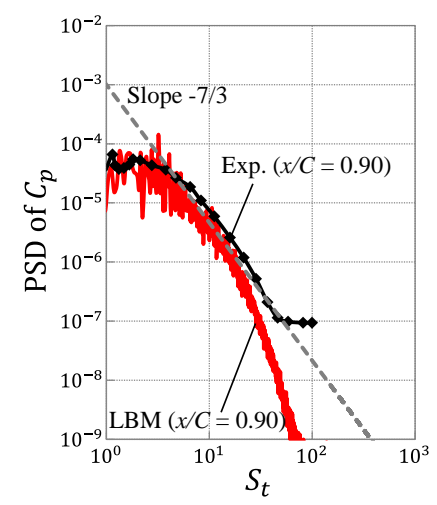

(c)

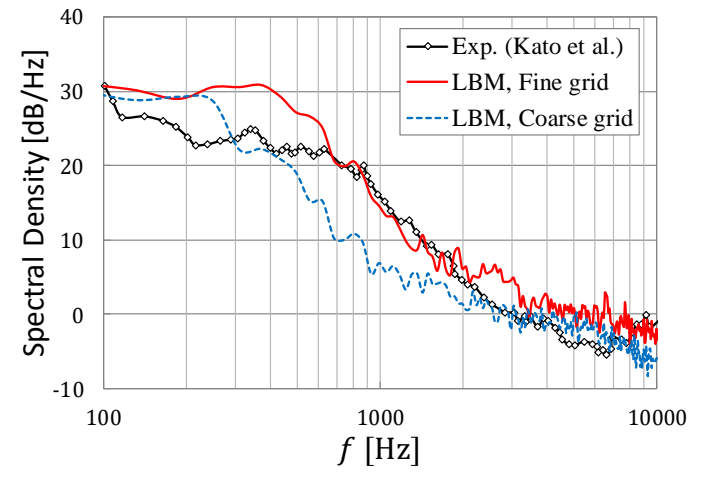

Fig. 20. Frequency spectra of far-field sound. 


\section{Conclusions}

The numerical method based on the LBM with wall-resolved grid was proposed to predict the broadband sound generated from attached turbulent flows at low Mach numbers. In the method, the athermal models of D2Q9 and D3Q15 were adopted to simulate weakly compressible flows at low Mach numbers with minimal computational cost. Furthermore, a multi-scale approach using hierarchically refined grids was proposed to efficiently and simultaneously capture the multi-scale phenomena of turbulent eddies near walls and far-field sound waves. Numerical instabilities caused by the lack of grid resolution were suppressed with a fourth-order implicit filtering scheme. The proposed method was tested with two benchmark problems and applied to the prediction of the broadband sound generated from an attached turbulent flow around an isolated airfoil.

First, the computational accuracy and speed of the LBM were assessed with a pulse-propagating problem. The LBM can achieve accuracy comparable to the fourth-order central scheme with the fourstage Runge-Kutta method for the compressible Navier-Stokes (N-S) equations and compute 12.3 times faster than the N-S simulation. These findings suggest that the LBM is an efficient computational method for aeroacoustic simulations.

Second, the proposed method was validated by simulating the Aeolian tone generated by the flow past a circular cylinder at Reynolds number of 150 and Mach number 0.2. The present simulation was in good agreement with the high-order finite difference scheme for the compressible N-S equations in terms of the wave profile and the propagation speed of the tonal sound. This validation results suggest that the present method based on athermal modeling is available for direct aeroacoustic simulations of low-Mach-number flows.

Finally, the capability of the present method to predict the broadband sound was demonstrated by conducting a wall-resolved simulation for the turbulent flow generated by a short separation bubble over an isolated airfoil at Reynolds number of $2.0 \times 10^{5}$ and Mach number 0.058 . This simulation was in good agreement with measurements of the surface pressure distributions, the wake velocity profiles, and the far-field sound spectrum. In contrast to the conventional hybrid approaches based on the incompressible N-S equations, the present method can simulate the acoustic scattering of highfrequency sounds generated from the turbulent flow on the airfoil. Consequently, the method successfully predicted the broadband sound spectrum in high-frequency range.

\section{Acknowledgment}

This work was supported by Japan Society for the Promotion of Science (JSPS), Grant-in-Aid for JSPS Fellow, No. 12J06308, 2012-2013. 


\section{Appendix A: Computational domain size}

This appendix shows the influence of the computational domain size on the sound prediction for an isolated airfoil presented in the section 4. Two different sized computational domains, Domain 1 and Domain 2, shown in Fig. A.1 were investigated. The size of Domain 1 is $24 C \times 24 C \times 0.125 C$, which was used in the section 4 . The size of Domain 2 is $48 C \times 48 C \times 0.125 C$, which is two times larger than Domain 1 in $x$ and $y$ directions. The absorbing region was set at $r>8 C$ and $r>16 C$ for Domain 1 and Domain 2, respectively. The width of the absorbing region $L_{d}$ is $4 C$ in the both domains.

Figure A.2 shows the frequency spectra of the far-field sound observed at a position of $6.7 C$ away from the airfoil in the vertical direction. Although the spectrum of Domain 2 is not smooth as that of Domain 1 due to short computational duration, these results are almost the same level. These results indicate that Domain 1 is sufficiently large for the sound prediction. Furthermore, these results suggest that the absorbing region was able to prevented sound reflections; otherwise the spectra of the both results would indicate different levels due to differences in the distance decay.

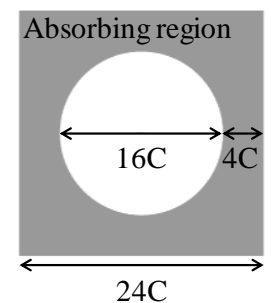

(a)

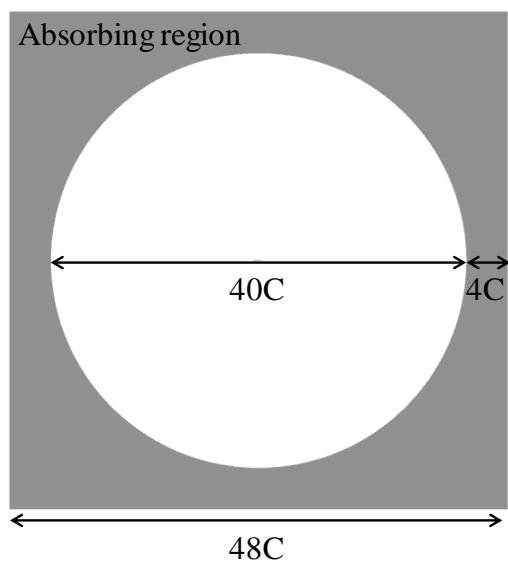

(b)

Fig. A.1. Computational domains. (a) Domain 1; (b) Domain 2.

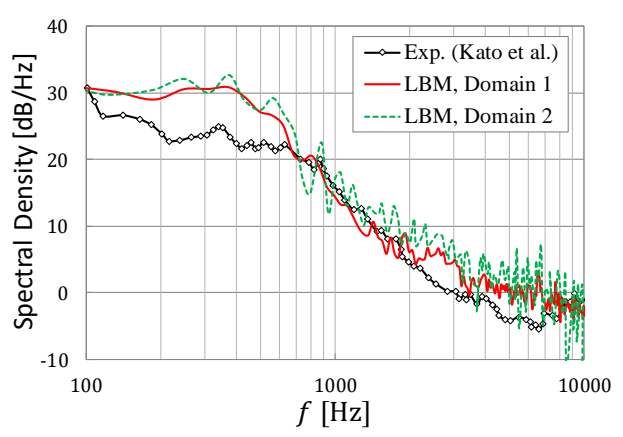

Fig. A.2. Frequency spectra of far-field sound. 


\section{Appendix B: SPL correction with respect to span length}

In Section 4, the periodic boundary condition was employed in the spanwise direction, and as a result, the sound radiated from the airfoil with the infinite span length was simulated. The computed SPL is required to be corrected based on the airfoil span length in accordance with the measurement condition. This correction was performed in two steps. First, the SPL in the computational span length was estimated from the SPL computed in the infinite span length using the method shown in the following. Second, the SPL in the computational span length was corrected to the SPL in the experimental span length using the method proposed by Kato et al. [40, 41].

The first SPL correction from the infinite span length to the computational domain length is derived as follows. The sound source is simplified as a point row uniformly distributed on the z-axis with $I$ acoustic energy per unit length as shown in Fig. B.1. Considering the distance decay, the acoustic energy $I_{\infty}$ radiated from the infinite row of point sound sources to an observer at $z=0, y=R$ is expressed as follows:

$$
I_{\infty}=\frac{1}{4 \pi} \lim _{L \rightarrow \infty} \int_{-L}^{L} \frac{I}{R^{2}+z^{2}} \mathrm{~d} z=\frac{I}{4 \pi}
$$

In addition, the acoustic energy $I_{c}$ radiated from the computational span length $L_{c}$, which is assumed to be sufficiently small compared with the distance $R\left(L_{c} \ll R\right)$, to the observer is calculated as

$$
I_{c}=\frac{L_{c} I}{4 \pi R^{2}}
$$

By multiplying the ratio of $I_{c} / I_{\infty}$ with the directly computed acoustic energy, the SPL correction formula is

$$
\mathrm{SPL}_{c}=\mathrm{SPL}_{\infty}+10 \log _{10}\left(\frac{L_{c}}{\pi R}\right)
$$

where $\mathrm{SPL}_{\infty}$ indicates the SPL computed under the condition of the infinite span length, and $\mathrm{SPL}_{c}$ indicates the SPL corrected to the computational span length.

In the second step, the SPL in the experimental span length is calculated from the smaller computational span length using a method based on the spanwise coherence length. Refer to the literature for details $[40,41]$.

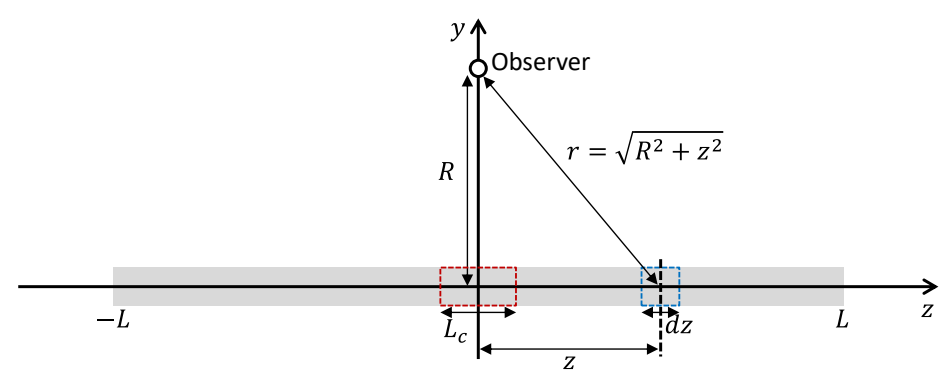

Fig. B.1. The calculation model of the sound radiated from an infinite row of point sound sources. 


\section{References}

[1] V. Jurdic, P.F. Joseph, A. Moreau, L. Enghardt, Predictions of fan broadband noise due to rotor-stator interaction, 14th International Congress on Sound and Vibration (2007).

[2] H. Reese, T. Carolus, C. Kato, Numerical prediction of the aeroacoustic sound sources in a low pressure axial fan with inflow distortion, Fan Noise 2007 (2007).

[3] T. Zhu, D. Lallier-Daniels, M. Sanjose, S. Moreau, T. Carolus, Rotating coherent flow structures as a source for narrowband tip clearance noise from axial fans, J. Sound Vib. 417 (2018) 198-215.

[4] M. Wang, P. Moin, Computation of trailing-edge flow and noise using large-eddy simulation, AIAA J. 38 (2000) 2201-2209.

[5] C. Kato, Y. Yamade., H. Wang, Y. Guo, M. Miyazawa, T. Takaishi, S. Yoshimura, Y. Takano, Numerical prediction of sound generated from flows with a low Mach number, J. Comput. Fluids 36 (2007) 53-68.

[6] M.J. Lighthill, On sound generated aerodynamically I. General theory, Proc. Royal Soc. Lond. A, Vol.211, No.1107 (1952), pp.564-587.

[7] N. Curle, The influence of solid boundaries upon aerodynamic sound, Proc. Royal Soc. Lond. A, Vol.231, No.1187 (1955), pp.505-514.

[8] Y.H. Choi, C.L. Merkle, The application of preconditioning in viscous flows, J. Comput. Phys. 105 (1993) 207-223.

[9] S. Marie, D. Ricot, P. Sagut, Comparison between lattice Boltzmann method and Navier-Stokes high order schemes for computational aeroacoustics, J. Comput. Phys. 228 (2009) 1056-1070.

[10] M. Tsutahara, The finite-difference lattice Boltzmann method and its application in computational aero-acoustics, Fluid Dyn. Res. 44 (2012).

[11] A.T. Jong, H. Bijl, A. Hazir, J. Wiedemann, Aeroacoustic simulation of slender partially covered cavities using a lattice Boltzmann method, J. Sound Vib. 332 (2013) 1687-1703.

[12] G.A. Bres, D. Freed, M. Wessels, S. Noelting, F. Perot, Flow and noise predictions for the tandem cylinder aeroacoustic benchmark, Phys. Fluids 24 (2012) 036101.

[13] Avallone, F., van der Velden, W., Ragni, D. and Casalino, D., Noise Reduction Mechanisms of Sawtooth and Combed-Sawtooth Trailing-Edge Serrations, J. Fluid Mech., Vol. 848, pp. 560-591, 2018.

[14] Casalino, D., Hazir, A., and Mann, A., Turbofan Broadband Noise Prediction using the Lattice Boltzmann Method, AIAA J., Vol. 56, No. 2 (2018), pp. 609-628.

[15] Romani, G., and Casalino, D., Rotorcraft Blade-Vortex Interaction Noise Prediction using the LatticeBoltzmann Method, Aerospace Science and Technology, Vol. 800, pp. 147-157, 2019.

[16] Ribeiro, A. F. P, Casalino, D., Fares, E. and Choudari, M., Direct Numerical Simulation of an Airfoil with Sand Grain Roughness on the Leading Edge, NASA/TM-2016-219363, 2017.

[17] H. Chen, S. Chen, W.H. Matthaeus, Recovery of the Navier-Stokes equations using a lattice-gas Boltzmann method, Phys. Rev. A 45 (1992).

[18] Y.H. Qian, D. d'Humieres, P. Lallemand, Lattice BGK Models for Navier-Stokes Equation, Europhys. Lett. 17 (1992) 479-484.

[19] J.M. Buick, C.A. Greated, D.M. Campbell, Lattice BGK simulation of sound waves, Europhys. Lett. 43 (1998) 235-240.

[20] T. Krueger, H. Kusumaatmaja, A. Kuzmin, O. Shard, G. Silva, E. M. Viggen, The Lattice Boltzmann Method- Principles and Practice. Berlin, Switzerland: Springer, 2017.

[21] J. Latt, B. Chopard, O. Malaspinas, M. Deville, A. Michler, Straight velocity boundaries in the lattice Boltzmann method, Phys. Rev. E 77(5), 056703, 2008.

[22] K. Nakahashi, L.S. Kim, Building-cube method for large-scale, high resolution computations, Proceedings of the $42^{\text {nd }}$ AIAA Aerospace Science Meeting and Exhibit (2004), pp.676-684.

[23] O. Filippova, D. Hanel, Grid refinement for lattice-BGK models, J. Comput. Phys. 147 (1998) 219- 
228.

[24] S. Succi, O. Filippova, H. Chen, S. Orszag, Towards a renormalized lattice Boltzmann equation for fluid turbulence, J. Stat. Phys. 107 (2002) 261-282.

[25] D.V. Gaitonde, M.R. Visbal, Pade-Type higher-order boundary filters for the Navier-Stokes equations, AIAA J. 38 (2000) 2103-2112.

[26] M. Bouzidi, M. Firdaouss, P. Lallemand, Momentum transfer of a Boltzmann-lattice fluid with boundaries, Phys. Fluids 13, (2001) 3452-3459.

[27] S. Kam, E.W., C. So, R. M., K. R.C. Leung, Lattice Boltzmann method simulation of aeroacoustics and nonreflecting boundary condition, AIAA J. 45 (2007) 1703-1712.

[28] S. Lele, Compact finite difference schemes with spectral-like resolution, J. Comput. Phys. 103 (1992) 16-42.

[29] Christopher K. W. Tam and Jay C. Webb, Dispersion-Relation-Preserving Finite Difference Schemes for Computational Acoustics, J. Comput. Phys. 107 (1993) 262-281.

[30] J. D. Sterling and S. Chen, Stability Analysis of Lattice Boltzmann Methods, J. Comput. Phys. 123 (1996) 196-206.

[31] O. Inoue, N. Hatakeyama, Sound generation by a two-dimensional circular cylinder in a uniform flow, J. Fluid Mech. 471 (2002) 285-314.

[32] Cimbala, J. M., Nagib, H. M., Roshko, A., Large structure in the far wakes of two-dimensional bluff bodies, J. Fluid Mech. 190 (1988), 265-298.

[33] M. Miyazawa, C. Kato, Y. Suzuki, T. Takaishi, Aeroacoustic simulation of a flow around a 2-D aerofoil (1st report, validation of a large eddy simulation of separated and transitional flow around an aerofoil), J. Jpn. Soc. Mech. Eng. 72 (2006) 58-65 (in Japanese).

[34] O. Marsden, C. Bogey, C. Bailly, Direct noise computation of the turbulent flow around a zeroincidence airfoil, AIAA J. 46 (2008) 874-883.

[35] H. Shan, L. Jiang, C. Liu, M. Love, B. Maines, Numerical study of passive and active flow separation control over a NACA0012 airfoil, J. Comput. Fluids 37 (2008) 975-992.

[36] W.R. Wolf, J.L.F. Azevede, S.K. Lele, Convective effects and the role of quadrupole sources for aerofoil aeroaoustics, J. Fluid Mech. 708 (2012), 502-538.

[37] W. Zhang, R. Samtaney, Assessment of spanwise domain size effect on the transitional flow past an airfoil, J. Comput. Fluids 124 (2016), 39-53.

[38] H.P. Horton, Laminar separation in two and three-dimensional incompressible flow, PhD Dissertation, University of London, (1968).

[39] M. Alam, N.D. Sandham, Direct numerical simulation of 'short' laminar separation bubbles with turbulent reattachment, J. Fluid Mech. 410 (2000) 1-28.

[40] C. Kato, M. Ikegawa, Large eddy simulation of unsteady turbulent wake of a circular cylinder using the finite element method, Advances in Numerical Simulation of Turbulent Flows; Proceedings of the Symposium, ASME and JSME Joint Fluids Engineering Conference, (1991), pp.49-56.

[41] C. Kato, Y. Takano, A. Iida, H. Fujita, M. Ikegawa Numerical prediction of aerodynamic sound by large eddy simulation (1st report, aerodynamic sound radiated from two-dimensional circular cylinder), J. Jpn Soc. Mech. Eng. 60 (1994) 126-132 (in Japanese). 